\title{
Networked biomedical system for ubiquitous health monitoring
}

\author{
Arjan Durresi ${ }^{\mathrm{a}, *}$, Mimoza Durresi $^{\mathrm{a}}$, Arben Merkoci ${ }^{\mathrm{b}}$ and Leonard Barolli ${ }^{\mathrm{c}}$ \\ ${ }^{a}$ Department of Computer and Information Science, Indiana University Purdue University \\ Indianapolsi, Indianapolis, IN 46202, USA \\ ${ }^{\mathrm{b}}$ Departament de Quimica, Universitat Autònoma de Barcelona, Barcelona, Spain \\ ${ }^{\mathrm{c}}$ Department of Information and Communication Engineering, Fukuoka Institute of Technology, \\ Fukuoka, 811-0295, Japan
}

\begin{abstract}
We propose a distributed system that enables global and ubiquitous health monitoring of patients. The biomedical data will be collected by wearable health diagnostic devices, which will include various types of sensors and will be transmitted towards the corresponding Health Monitoring Centers. The permanent medical data of patients will be kept in the corresponding Home Data Bases, while the measured biomedical data will be sent to the Visitor Health Monitor Center and Visitor Data Base that serves the area of present location of the patient. By combining the measured biomedical data and the permanent medical data, Health Medical Centers will be able to coordinate the needed actions and help the local medical teams to make quickly the best decisions that could be crucial for the patient health, and that can reduce the cost of health service.
\end{abstract}

Keywords: Heterogeneous networks, wireless communications, sensors, health monitoring

\section{Introduction}

The aging of populations is becoming a social and economical challenge worldwide. For example, according to [19], "In the United States alone, the number of people over age 65 is expected to hit 70 million by 2030, doubling from 35 million in 2000, and similar increases are expected worldwide." On the other hand, many elderly people suffer from chronic diseases that require medication and clinic visits on a regular basis. Therefore, for this growing category of people, it is crucial to monitor the health condition all the time. Health monitoring can save lives, improve the quality of life for many, and last but not least, it can reduce the cost of health care, by enabling early interventions. The cost of the health care is a growing problem too, for example, expenditures in the United States for healthcare will grow to $15.9 \%$ of the GDP ( $\$ 2.6$ trillion) by 2010 (Digital 4Sight's Healthcare Industry Study) as a result of the accumulative impact of chronic degenerative diseases in the elderly and their increasing dependence on the health care system.

Next generation networked medical devices and health management systems are envisioned to be ubiquitous systems of networked systems for secure, reliable, privacy-preserving, cost-effective and personalized quality health care, leading not only to better health-care delivery, but also to improving people's quality of life in general. Unfortunately, current medical devices are still mostly standalone

\footnotetext{
${ }^{*}$ Corresponding author. Tel.: +1 3172748942; Fax: +1 3172749742; E-mail: durresi@cs.iupui.edu.
} 
subsystems with proprietary designs. Medical workers often need to manually transfer data among several machines.

There are various research projects going on in the direction of using sensor and network technologies for health services. The closest work to our solution is I-Living [1,21], an assisted-living supportive system, being developed by researchers at the University of Illinois at Urbana-Champaign. The major goal of I-Living is to assist elderly people to get the needed health services, while staying at home.

At the Center for Future Health (CFH) at University of Rochester [7] the smart medical home prototype consists of infrared sensors, computers, bio-sensors, and video cameras. The key services to be provided are medical advisory, which provides a natural conversational interface between the patient and health care expert, motion and activity monitoring, pathogen detection and skin care, and personal health care recording for consumer-provider decision support.

The Aware Home project at Georgia Tech [3] targets to create a home environment that is aware of its occupants' whereabouts and activities. The services provided by Aware Home range from enhancing social communications such as providing digital portrait of elderly people to their family members, to memory aids that assist users in resuming interrupted activities based on playbacks of past events recorded by video camera.

The smart in-home monitoring system at University of Virginia [8] focuses on data collection with the use of a suite of low-cost, non-intrusive sensors. The information collected is logged and analyzed in an integrated data management system (that is linked to the Internet). The system essentially collects information in a passive manner and does not directly interact with elderly people.

The major industry research effort is perhaps led by the age-in-place advanced smart-home system at Intel [4]. It aims to help elderly people with Alzheimer's diseases, by integrating four major technologies: sensors, home networks, activity tracking, and ambient displays. The sensors located in the home environment sense the locations of the people and the objects in the home.

Differently from the above mentioned strategies, our goal is to provide ubiquitous health monitoring, at home, and outside it, all the time. People need to have their health conditions under control not only when they are at home, but everywhere. In our technological society, we are proud of our ubiquitous communication or computing capabilities, but we think, it would be a point of even higher proud, if we could have ubiquitous health monitoring, which would improve the life quality for many. If our technology is not used to improve people's life, what is it good for?

We define the following requirements for our architecture:

1. Reliability: Due to the sensitive nature of our system, it should work even when various components might fail. For example, to guarantee the connectivity of the patients to the system, we propose to use complementary multiple wireless technologies.

2. Quality of service: The system should provide high Quality of Service. For this reason, our communication protocols and our information management systems have to be designed and dimensioned with this requirement in mind.

3. Security and privacy: Are a must for the whole system. The system will guarantee confidentiality, integrity, authentication, and privacy of data.

The rest of the paper is organized as follows. Section 2 provides an overview of our system architecture. In Section 3 we discuss the communication architecture. Section 4 presents our information management and security architecture. We conclude in Section 5. 


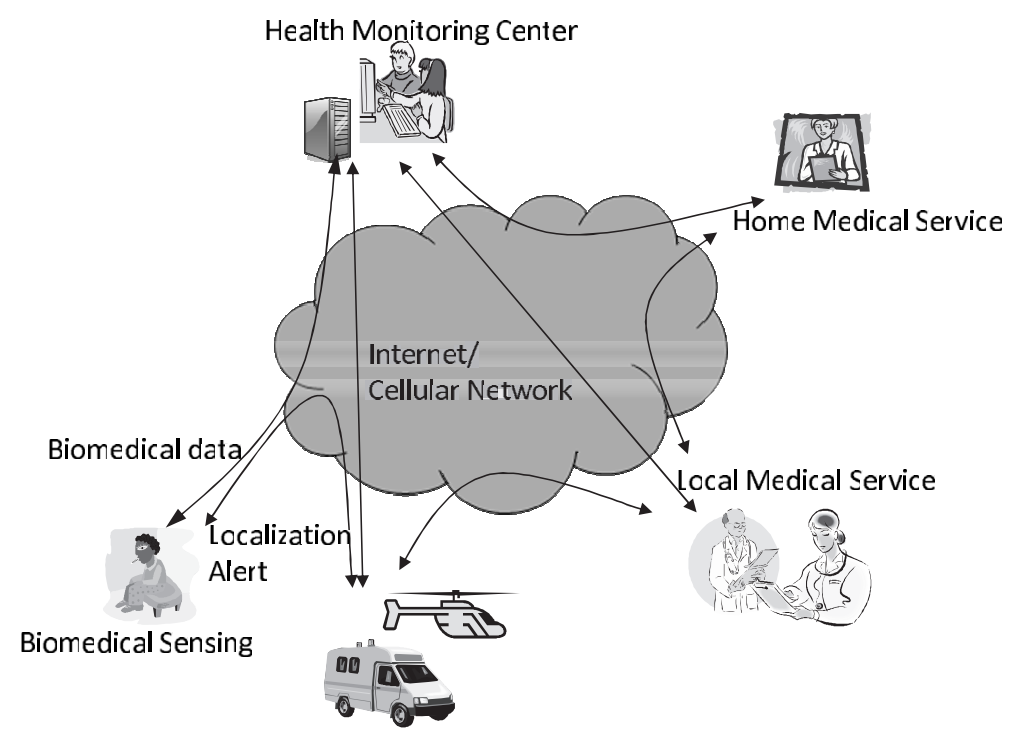

Fig. 1. System architecture. Patient's biomedical data will be sent via multiple wireless networks to the appropriate Health Monitor Center, which will coordinate the activities among the Home and Local Medical Services and various emergency services.

\section{Architecture overview}

In Fig. 1 we show an overview of the architecture that we are proposing for the Ubiquitous Health Monitoring System.

The biomedical data will be generated by multiple sensors that can be integrated in devices wearable by patients, such as necklaces, bracelets, etc. Sensors could include Carotid Dopplers, ECG Nanotrodes, biochip based on tissue/fluids Quantum Dots, etc. The measured biomedical data could include vital signs, such as the glucose level, blood pressure, heart bit rate, arterial oxyhemoglobin saturation level, etc.

The measured biomedical data will be transmitted via multiple complementary wireless networks, through the Internet, towards the appropriate Health Monitoring Center (HMC), where this data will be integrated with the permanent medical data of the given patient. Therefore, the medical personnel at HMC will be able to monitor various vital signs at desirable time granularity. Should the readings suggest any abnormal health situations, medical instructions can be given and actions can be taken before the situations deteriorate.

If needed, the personnel at HMC can consult with the patient's personal doctor, whose information is kept at Home Medical Center. Depending on the situation, HMC can coordinate the immediate medical service in the closest or more appropriate local medical facility using the best transportation service available. HMC can also coordinate a quick location discovery of the patient, in order to minimize the delays in providing the medical help, delays that in many cases make the difference between life and death.

Our goal is to monitor the patient's health everywhere, in their homes and outside. Therefore, we will distinguish two typical scenarios: (1) When the patient is at home or in the surrounding area; and (2) When the patient is far away from his home, for example in another city. To cover both cases, we propose a distributed health monitoring system. Therefore, the Health Monitoring Center functionalities will be distributed over the territory. In case (1), when the patient is at home, his/her measured biomedical data 
will be sent to the Home Health Monitoring Center. On the other hand, in case (2), when the patient is in another location, far away from his/her home, the measured biomedical data will be sent to the corresponding Visitor Health Monitoring Center.

In Section 4, we discuss in more details the level of distribution of $\mathrm{HMC}$ and capacity planning issues of the system.

\section{Communication architecture}

While there are various communication components in our architecture, as shown in Fig. 1, we will focus in this paper on the access communication architecture, which is between the patient and the Internet, as the most critical part of communications involved in the whole system. Again, we will distinguish two cases (1) when the patient is home and (2) when he/she is far away from home.

\subsection{Home health monitoring}

In this case, the local access network could be based on IEEE 802.11 family of wireless protocols. The biomedical data, measured by various sensors, will be sent using wireless Bluetooth networking to a wearable communication/computing device, such as a PDA or cell phone, which will have special software to treat the measured information, to filter, merge, store, and finally sent it to an Access Point (AP) (inside the house) using an IEEE 802.11 wireless interface. We assume that AP are connected to the Internet, therefore, the biomedical data, will go through Internet to the Home Health Medical Center.

To guarantee high communication reliability, we propose to use multiple complementary wireless technologies to provide connectivity among the patients and the system. Therefore, while IEEE 802.11 could be, as explained above, one way of network access, other solutions should be investigated and possibly be used as alternative interface. For example, the patient wearable communication/computing device could be equipped, beside the IEEE 802.11 interface, with cellular phone, IEEE 802.16, and other wireless interfaces, as shown in Fig. 2. So when the patient is out of the reach of his/her IEEE 802.11 network, the data will be sent via cellular network, IEEE 802.16 or other interfaces. In case all such interfaces fail, which most likely will happen when the patient is not close to his home, we propose an ad hoc wireless network, which will be explained in more detail in Section 3.2.

\subsection{Mobile health monitoring}

When patients are away from their homes, again we propose multiple complementary wireless networks to be used as access network between the patient and the Internet.

In the case of mobile conditions, we propose cellular phone to be used as the first choice for the access network. The reason is that cellular phones are becoming ubiquitous telecommunication devices. Beside the low cost, their multitude of features, make cellular phones our most used personal communication devices. It is estimated that there are over 2 billion cell phones worldwide [2]. These phones typically have low power transceivers which typically transmit data and voice up to a few miles where the mobile tower (base station) is located. This base station connects the cellular phone to the backbone telephone network. The mobile phones cannot communicate when they are unable to connect to the base station.

The capabilities of these phones have also increased dramatically over the last few years. In addition to the standard telephone features, the phones also Instant Messaging, MMS, Internet access etc. More advanced features like music and video streaming, digital camera, and document scanner are being 


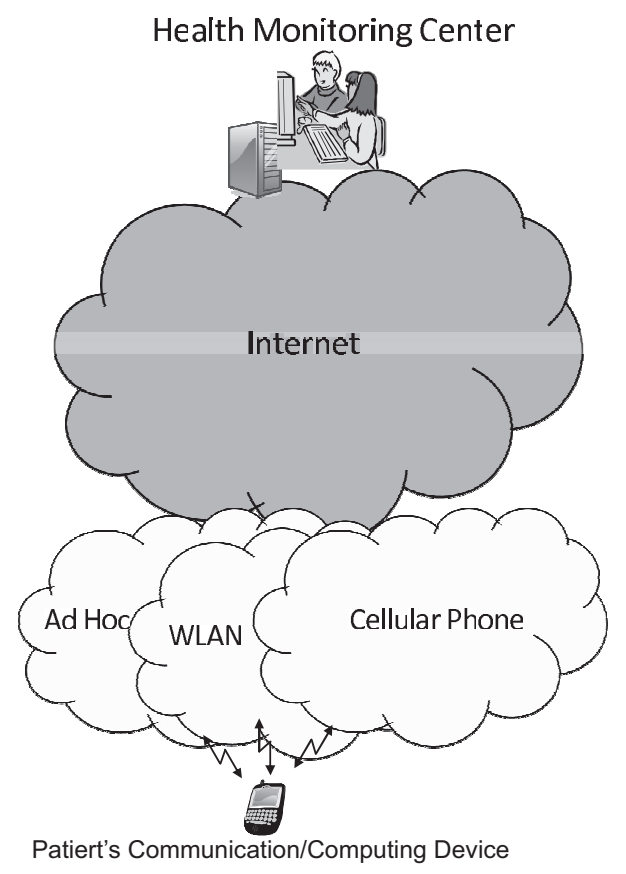

Fig. 2. The patient communication device, after collecting the measured biomedical data from various sensors, will communicate using multiple wireless networks with the Health Monitoring Center via Internet.

bundled with the cell phone. These features have transformed the cell phone from a simple phone to a digital Swiss army knife.

More advanced features like bluetooth, IR have been added to allow the cell phone to connect with other devices. Avaya, Motorola, and Proxim are planning to introduce a new class of mobile phones called dual phones [5,6], also referred to as the dual phones. These phones will be able to make voice calls over the cellular network and the 802.11a WLAN networks. The advantage of using this phone is that the user can make calls through the WLAN infrastructure when he is able to connect to the WLAN. This would save money because the cell phone user would be able to use the WLAN minutes for free. The companies have also developed the technology to hand off calls between the WLAN and cellular network. Already there are on the market cell phones able to connect to both the cellular network and the wireless devices. Such an ability could allows them to be used in many applications, including our health monitoring system.

We would like to stress again that reliability is the key distinguishing characteristic of our proposed architecture. Therefore, one important case is that when neither cellular phone system nor various WLAN technologies are available to patients in specific locations. For such cases we propose to use cell phones as ad hoc devices. As a consequence, existing cell phones in the area will create an ad hoc network (using similar interfaces such as IEEE 802.11) and will forward messages (containing biomedical data) even when the cellular infrastructure is not working.

We have developed routing $[13,14]$ and broadcast protocols $[9,17,18]$ to be used over ad hoc networks of cell phones. For example, our Adaptive Geometric Broadcast Protocol (AGB) for Heterogeneous Wireless Ad Hoc Networks $[12,17,18]$ starts with a geometric approach and adapts itself to real conditions to use at best the available resources. Consequently, AGB is simple, robust and very scalable. On the other hand, our Adaptive Routing and Energy Management (AREM) [10,13,14,20], an adaptive cosslayer 


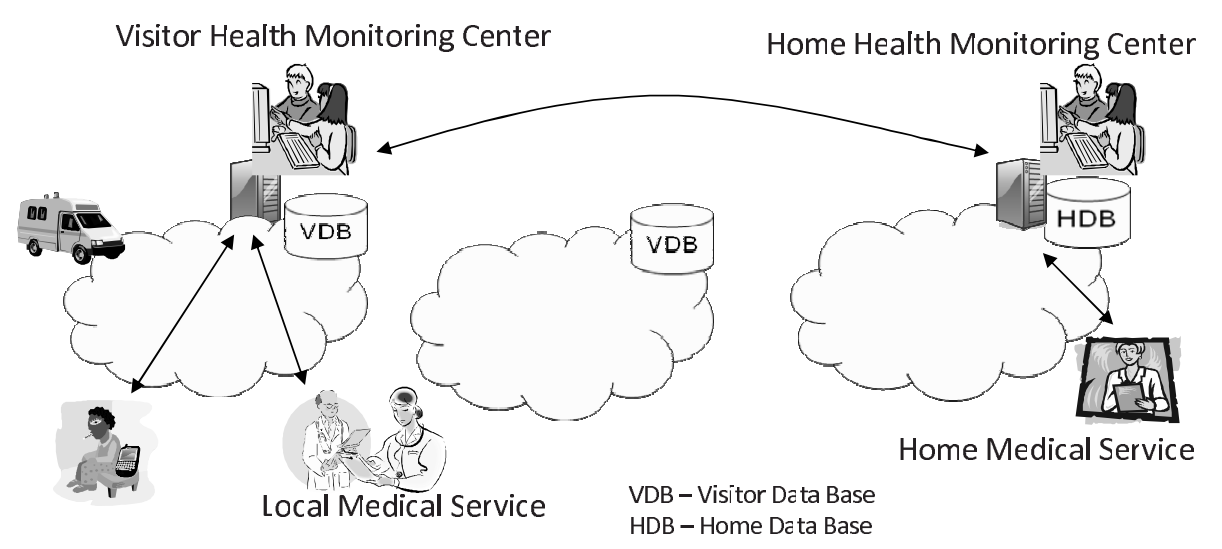

Fig. 3. The Health Monitoring Centers are distributed over the territory. Each patient is affiliated with a Home Health Monitoring Center, which keeps his/her permanent medical data. The patient will be served by the corresponding Visitor Health Monitoring Center (if he/she is not in his area), which in collaboration the his/her Home Health Monitoring Center will coordinate the activities among the Local and Home Medical service.

communication protocol, dynamically adapts the transmission ranges to minimize the delay and the energy consumption, and to prolong both node and network lifetimes. For the particular case when patients are traveling on highways, we have proposed another intervehicle communication protocol [11] that can be used to connect patients to the health monitoring system.

\section{Information management and security architecture}

The information management architecture is shown in Fig. 3. The territory is covered by distributed Health Monitoring Centers. Each patient will be affiliated with a Home Health Monitoring Center (HHMC), which has a Home Data Base (HDB), where the permanent medical data of the given patient are stored. The permanent medical data are periodically updated from the corresponding Home Medical Service (personal doctor).

When the patient is located inside the coverage area of his/her HHMC, the measured biomedical data will be sent to this center, to be merged there with his/her permanent medical data. Then, the personnel of HHMC, in collaboration with that of the patient's Home Medical Service, will coordinate the needed actions.

When the patient is located far away from his home, he/she will be covered by a given Visitor Health Monitor Center (VHMC), and the measured biomedical data will be stored in the Visitor Data Base (VDB) of this center. When needed, the VHMC will contact the HHMC to retrieve the permanent medical data of the patient from the HDB, update the VDB, and merge them with the measured biomedical data. Then, the personnel of VHMC will coordinate the activities among the Home Medical Service and the Local Medical Service, to provide the best medical help to the patient.

One important aspect of the Information management System is its capacity dimensioning. When Visitor Health Monitoring Centers cover large areas, the load work for each one of them could be high too. On the other hand, small coverage areas of Visitor Health Monitoring Centers, would distribute the load among them, but would increase the coordination load among them. Therefore, a careful tradeoff dimensioning of the system is needed, to provide the best and quickest service to the user.

All information flows in the system should be secure. We propose that before each exchange of information between two parts, cryptography authentication should take place first. So, the patient and 
the given HHMC or VHMC should authenticate each other. The same should happen between HHMC and VHMC, HMC and (Local of Home) Medical Service. Therefore, we assume that all parties share the needed keys among them. Using session keys, all exchanges of data will be end-to-end encrypted to guarantee the required confidentiality. Also, all exchanges of information will be protected from integrity point of view, with the appropriate digital signatures. Due to its delicate nature, the system will guarantee the privacy of biomedical data. Only authorized medical personnel will have access on the data and when it is needed. Solutions such as we have proposed in $[15,16]$ could be used for such purpose.

\section{Conclusions}

We propose a new architecture for a Ubiquitous Health Monitoring System. Our architecture is based on multiple complementary wireless communication access networks between the patient and the system, through the Internet. The biomedical data will be generated continually by wearable sensing devices. We propose to have a distributed Health Monitoring System. Each patient will be affiliated with e Home Health Monitoring Center. The territory will be covered by Visitor Health Monitoring Centers, which in collaboration with the Home Centers will provide the needed coordination among the Home and Local Medical Services, to quickly respond to any medical situation in the best and secure way.

\section{References}

[1] Assited Living Project Web page at University of Illinois at Urbana-Champaign. [Online]. Available: http://lion.cs. uiuc.edu/assistedliving/index.html.

[2] Cellularonline, http://www.cellular.co.za.

[3] Georgia Institute of Technology, Aware Home. [Online]. Available: http://www.cc.gatech.edu/fce/ahri/.

[4] Intel Corporation, Age-in-Place. [Online]. Available: http://www.intel.com/research/prohealth/cs-aging in place.htm.

[5] http://www.networkworld.com/news/2004/072604avaya.html.

[6] http://www.pcworld.com/news/article/0,aid,116334,00.asp.

[7] University of Rochester, Center of Future Health. [Online]. Available: http://www.futurehealth.rochester.edu/news/.

[8] University of Virginia, Smart In-Home Monitoring System. [Online]. Available: http://marc.med.virginia.edu/projects smarthomemonitor.html.

[9] A. Durresi and V. Paruchuri, Broadcast Protocol for Energy-Constrained Networks, IEEE Transaction on Broadcasting 53 (2007), 112-119.

[10] A. Durresi, Architectures for Heterogeneous Wireless Sensor Networks, Proceedings of the 16th Annual IEEE International Symposium on Personal Indoor and Mobile Radio Communications - PIMRC'2005. Berlin, Germany, September 11-14, 2005, Invited paper.

[11] M. Durresi, A. Durresi and L. Barolli, Adaptive Intervehicle Communications, International Journal of Wireless Information Networks, Springer Science 13(2) (April 2006), 151-160.

[12] A. Durresi and V. Paruchuri, Geometric Broadcast Protocol for Sensor and Actor Networks. Proceedings of IEEE AINA 2005, Taipei, Taiwan, March 27-29, 2005, 343-348.

[13] A. Durresi, V. Paruchuri and L. Barolli, Delay-Energy Aware Routing Protocol for Sensor and Actor Networks, Proceedings of the 11th IEEE International Conference on Parallel and Distributed Systems ICPADS'2005, Fukuoka, Japan, July 20-22, 2005, 292-298, Excellent Paper Award.

[14] A. Durresi, V. Paruchuri and L. Barolli, Delay-Energy Aware Routing Protocol for Heterogeneous Ad Hoc Networks, Journal of Interconnection Networks (JOIN) 7(1) (March 2006), 37-49.

[15] A. Durresi, V. Paruchuri, L. Barolli and R. Kannan, Anonymous Communication Protocol for Sensor Networks, International Journal of Wireless and Mobile Computing (IJWMC), 2007, in press.

[16] A. Durresi, V. Paruchuri and S. Iyengar, A lightweight protocol for data integrity in sensor networks, Proceedings of The International Conference on Intelligent Sensors, Sensor Networks and Information Processing ISSNIP 2004, Melbourne, Australia, December 2004. 
[17] A. Durresi, V. Paruchuri, S. Iyegar and R. Kannan, Optimized Broadcast Protocol for Sensor Networks, IEEE Transaction on Computing 54(8) (2005), 1013-1024.

[18] A. Durresi, V. Paruchuri and R. Jain, Geometric Broadcast Protocol for Heterogeneous Wireless Sensor Networks, Journal of Interconnection Networks (JOIN) 6(3) (2005), 193-208.

[19] G.T. Huang, Monitoring Mom: As population matures, so do assisted-living technologies, Technical Review 20 (July 2003).

[20] V. Paruchuri, S. Basavaraju, A. Durresi, R. Kannan and S. Iyengar, Random Asynchronous Wakeup Protocol for Sensor Networks. Proceedings of BroadNets'04 San Jose, CA, October 2004, 710-717.

[21] Q. Wang, W. Shin, X. Liu, Z. Zeng, C. Oh, B.A. Li, M. Caccamo, C. Gunter, E. Gunter, J. Hou, K. Karahalios and L. Sha, I-Living: An Open System Architecture for Assisted Living. Proceedings of IEEE SMC, 2006. [Online] Available: http://lion.cs.uiuc.edu/assistedliving/publications/I-Living.pdf.

Arjan Durresi received his B.E., M.E. and Ph.D. (all summa cum laude) in Electronics and Telecommunications, in 1986, 1991 and 1993, respectively; and a Diploma of Superior Specialization in Telecommunications from La Sapienza University in Rome, Italy and Italian Telecommunications Institute. He is currently an Associate Professor in the Department of Computer and Information Science at Indiana University Purdue University at Indianapolis. Previously he held roles as Research Scientist of Computer Science and as Adjunct Professor of Electrical and Computer Engineering at The Ohio State University. His current research interests include network architectures, heterogeneous wireless networks, security, QoS routing protocols, traffic management, optical and satellite networks, and bioinformatics. Dr. Durresi has authored more than fifty journal papers, and more than eighty conference papers. He is an area editor for the Ad Hoc Networks Journal. He is the founder and cofounder of several workshops, including the IEEE International Workshops on Heterogeneous Wireless Networks - HWISE, the International Workshop on Advances in Information Security - WAIS, and the IEEE International Workshop on Bio Computing. $\mathrm{He}$ is a recipient of several paper awards in international conferences. Dr. Durresi has organized many international conferences and workshops. He is the co-chair of the 23rd IEEE AINA-2009.

Mimoza Durresi received the B.E. in Electronic-Telecommunications in 1989, a Diploma of Superior Specialization in Telecommunications from La Sapienza University in Rome, Italy and Italian Telecommunications Institute in 1991. She received the MS in Electric Computer Engineering in 2002 from The Ohio State University and the Ph.D. in Computer Engineering from Fukuoka Institute of Technology in 2006. She teaches at Indiana University Purdue University at Indianapolis. Previously she was with Franklin University. Her research interest are in wireless networking, inter vehicle communications and routing.

Arben Merkoci received is B.E. and Ph.D. from University of Tirana in 1986 and 1991 respectively. He is a Research Professor and Group Leader in the Institut Cata De Nanotecnologia, Spain. He is author of more than 150 referred journal and conference papers, 2 books, 5 book chapters and has one pending patent. He is winner of the Electrochemistry Communications Award 2005, in acknowledgment of high quality papers. He has been Editor of "Carbon nanotubes in Chemistry", Special Issue of Microchimica Acta, 2006 and Co-editor of "Analytical Nanoscience and Nanotechnology", Special Issue of Contribution to Science, 2005.

His research interest includes synthesis \& characterization of nanomaterials (Quantum Dots, carbon nanotube etc.) for their integration into electrochemical sensors and biosensors; electrochemistry and modern electroanalytical methods development and validation applied for nanostructures and other materials; and developing electrochemical biosensors based on DNA, antibodies and enzymes.

Leonard Barolli received B.E. and Ph.D. degrees from Tirana University and Yamagata University in 1989 and 1997, respectively. From April 1997 to March 1999, he was a JSPS Post Doctor Fellow Researcher at the Department of Electrical and Information Engineering, Yamagata University. From April 1999 to March 2002, he worked as a Research Associate at the Department of Public Policy and Social Studies, Yamagata University. From April 2002 to March 2003, he was an Assistant Professor at the Department of Computer Science, Saitama Institute of Technology (SIT). From April 2003 to March 2005, he was an Associate Professor and presently he is a Full Professor at Department of Information and Communication Engineering, Fukuoka Institute of Technology (FIT). Dr. Barolli has published more than 200 papers in referred Journals and International Conference proceedings. He was an Editor of the IPSJ Journal and has served as a Guest Editor for many International Journals. Dr. Barolli has been a PC Member of many International Conferences. He was the PC Chair of AINA-2004, ICPADS-2005, MNSA-2005 and NBiS-2005. He is General Co-Chair of AINA-2006 and MNSA-2006. His research interests include agentbased systems, distance learning, network traffic control, fuzzy control, genetic algorithms. He is member of SOFT, IPSJ, IEEE Computer Society and IEEE. Dr. Barolli have received may research awards and funded research grants. He received the appreciation certificate from IEEE Computer Society in 2004 and 2005. 

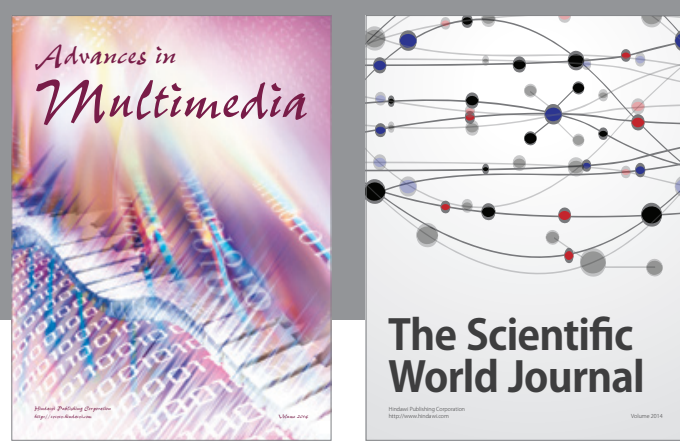

The Scientific World Journal
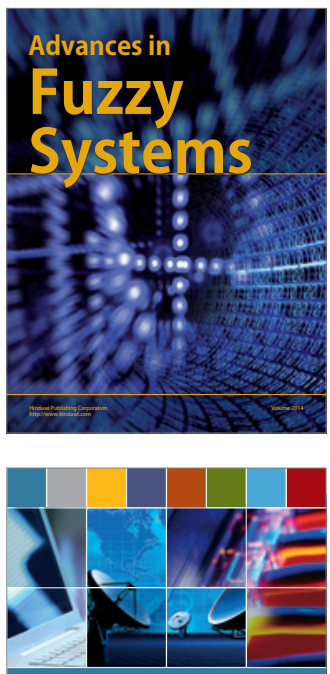

Computer Networks and Communications
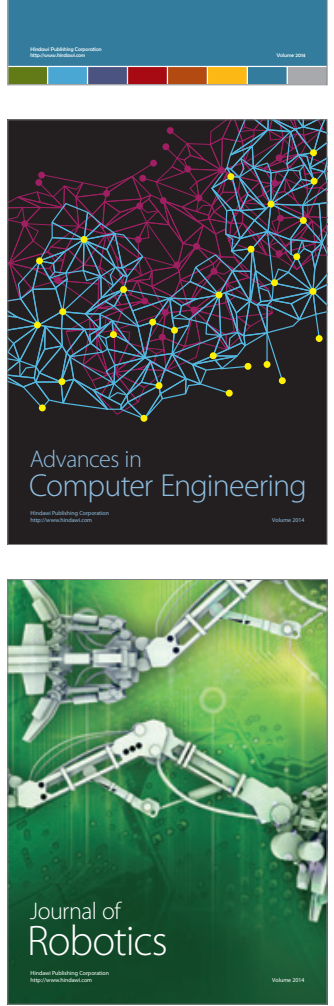
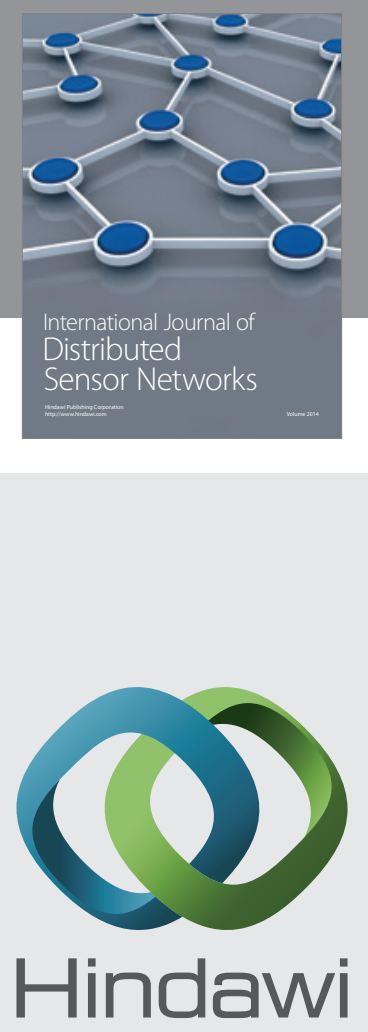

Submit your manuscripts at

http://www.hindawi.com
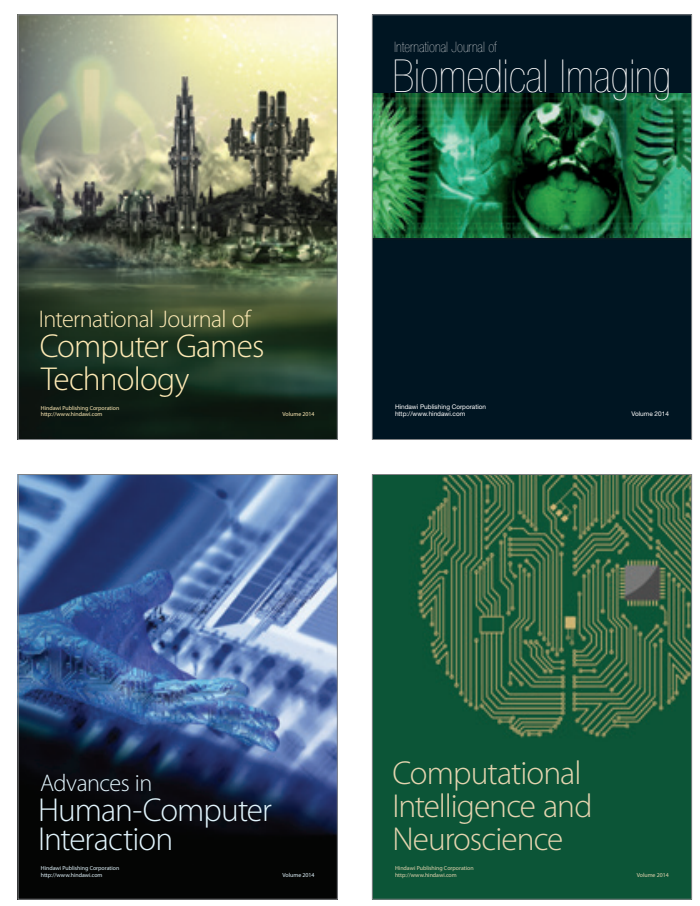
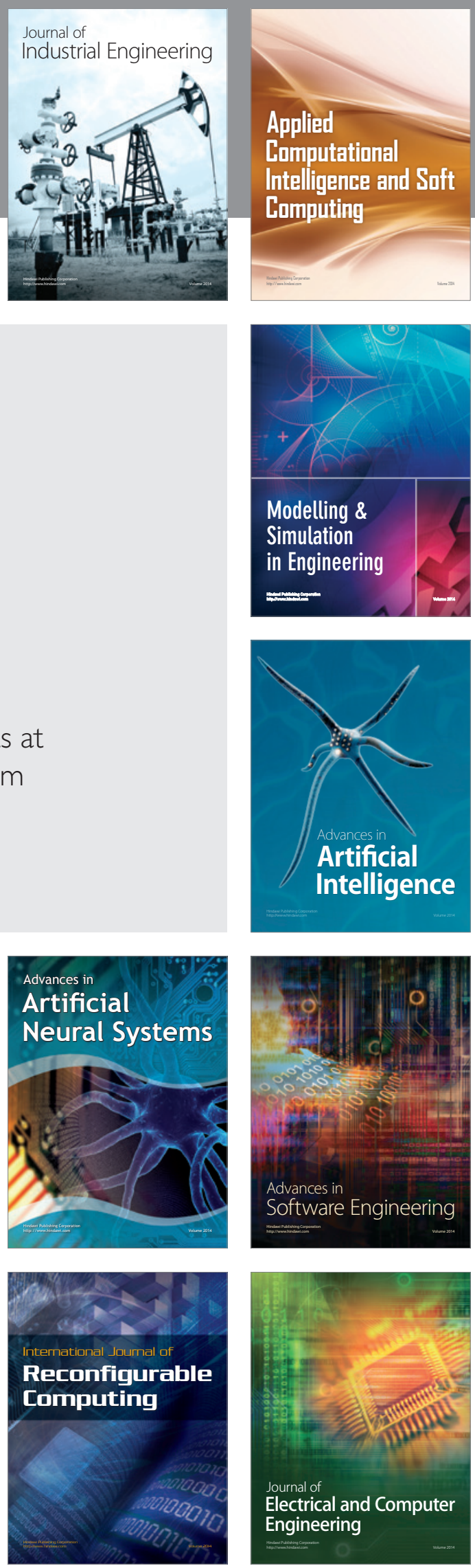